\title{
ON RELATIVE AND BI-RELATIVE ALGEBRAIC $K$-THEORY OF RINGS OF FINITE CHARACTERISTIC
}

\author{
THOMAS GEISSER AND LARS HESSELHOLT
}

\section{INTRODUCTION}

Throughout, we fix a prime number $p$ and consider unital associative rings in which $p$ is nilpotent. It was proved by Weibel [28, Cor. 5.3, Cor. 5.4] long ago that for such rings, the relative $K$-groups associated with a nilpotent extension and the bi-relative $K$-groups associated with a Milnor square are $p$-primary torsion groups. However, the question of whether these groups can contain a $p$-divisible torsion subgroup has remained an open and intractable problem. In this paper, we answer this question in the negative. In effect, we prove the stronger statement that the groups in question are always $p$-primary torsion groups of bounded exponent.

Let $A$ be a unital associative ring and let $I \subset A$ be a two-sided ideal. Then one defines the relative $K$-theory spectrum $K(A, I)$ to be the mapping fiber of the map of $K$-theory spectra $K(A) \rightarrow K(A / I)$ induced by the canonical projection. Hence, there is a long exact sequence of homotopy groups

$$
\cdots \rightarrow K_{q}(A, I) \rightarrow K_{q}(A) \rightarrow K_{q}(A / I) \stackrel{\partial}{\rightarrow} K_{q-1}(A, I) \rightarrow \cdots .
$$

Here and throughout, $K(A)$ denotes the non-connective Bass completed algebraic $K$-theory spectrum of the ring $A$ [26, Def. 6.4]. We prove the following result.

Theorem A. Let $A$ be a unital associative ring and $I \subset A$ a two-sided nilpotent ideal. Suppose that the prime number $p$ is nilpotent in $A$. Then for every integer $q$, the relative group $K_{q}(A, I)$ is a p-primary torsion group of bounded exponent.

It follows, in particular, from Theorem $\mathrm{A}$ that the $p$-completion map

$$
K_{q}(A, I) \rightarrow K_{q}\left(A, I ; \mathbb{Z}_{p}\right)
$$

is an isomorphism. We remark that, in general, the exponent of $K_{q}(A, I)$ depends on the degree $q$. For instance, if $A=\mathbb{F}_{p}[x] /\left(x^{m}\right)$ and $I=(x)$, the exponent tends to infinity with $q[19$, Thm. A].

In the situation of Theorem A, a theorem of McCarthy [23, Main Thm.] combined with a theorem of our own [11, Thm. 2.1.1] show that for a fixed positive integer $v$, the cyclotomic trace map of Bökstedt-Hsiang-Madsen [2] induces an isomorphism of pro-abelian groups

$$
K_{q}\left(A, I ; \mathbb{Z} / p^{v} \mathbb{Z}\right) \stackrel{\sim}{\rightarrow}\left\{\mathrm{TC}_{q}^{n}\left(A, I ; p, \mathbb{Z} / p^{v} \mathbb{Z}\right)\right\}
$$

Received by the editors February 18, 2009 and, in revised form, July 23, 2010.

2010 Mathematics Subject Classification. Primary 19D55; Secondary 18G50, 16S70.

The authors were supported in part by NSF Grant Nos. 0901021 and 0306519.

(C)2010 American Mathematical Society 
from the relative $K$-groups with $\mathbb{Z} / p^{v} \mathbb{Z}$-coefficients to the relative topological cyclic homology groups with $\mathbb{Z} / p^{v} \mathbb{Z}$-coefficients. By Theorem $\mathbb{A}$, we now conclude that the following stronger result holds.

Theorem B. Let $A$ be a unital associative ring and $I \subset A$ a two-sided nilpotent ideal. Suppose that the prime number $p$ is nilpotent in A. Then for every integer $q$, the cyclotomic trace map induces an isomorphism of pro-abelian groups

$$
K_{q}(A, I) \stackrel{\sim}{\longrightarrow}\left\{\mathrm{TC}_{q}^{n}(A, I ; p)\right\} .
$$

The pro-abelian group on the right-hand side is indexed by positive integers $n$.

We remark that Theorem $\mathrm{B}$ is equivalent to the statement that, for $n$ large, the group $K_{q}(A, I)$ embeds as a direct summand in $\operatorname{TC}_{q}^{n}(A, I ; p)$ and that the limit system of cokernels satisfies the Mittag-Leffler condition and has limit zero.

Let $f: A \rightarrow B$ be a map of unital associative rings and let $I$ be a two-sided ideal of $A$ that is mapped isomorphically onto a two-sided ideal of $B$. Then one defines the bi-relative $K$-theory spectrum $K(A, B, I)$ to be the mapping fiber of the map of relative $K$-theory spectra $K(A, I) \rightarrow K(B, f(I))$ induced by the map $f$. It follows that there is a long exact sequence of homotopy groups

$$
\cdots \rightarrow K_{q}(A, B, I) \rightarrow K_{q}(A, I) \rightarrow K_{q}(B, f(I)) \stackrel{\partial}{\rightarrow} K_{q-1}(A, B, I) \rightarrow \cdots .
$$

We prove the following result.

Theorem C. Let $f: A \rightarrow B$ be a map of unital associative rings, let $I \subset A$ be a two-sided ideal, and assume that $f: I \rightarrow f(I)$ is an isomorphism onto a two-sided ideal of $B$. Suppose that the prime number $p$ is nilpotent in $A$. Then for every integer $q$, the bi-relative group $K_{q}(A, B, I)$ is a p-primary torsion group of bounded exponent.

We remark again that Theorem $[$ implies that the completion map

$$
K_{q}(A, B, I) \rightarrow K_{q}\left(A, B, I ; \mathbb{Z}_{p}\right)
$$

is an isomorphism. The exponent of $K_{q}(A, B, I)$ in general depends on the degree $q$. For example, if $A=\mathbb{F}_{p}[x, y] /(x y), B=\mathbb{F}_{p}[x] \times \mathbb{F}_{p}[y]$, and $I=(x, y)$, the exponent tends to infinity with $q[17$, Thm. A].

In earlier work [10, Thm. 1], we have proved that for a fixed positive integer $v$, the cyclotomic trace map induces an isomorphism of pro-abelian groups

$$
K_{q}\left(A, B, I ; \mathbb{Z} / p^{v} \mathbb{Z}\right) \rightarrow\left\{\mathrm{TC}_{q}^{n}\left(A, B, I ; p, \mathbb{Z} / p^{v} \mathbb{Z}\right)\right\}
$$

from the bi-relative $K$-groups with $\mathbb{Z} / p^{v} \mathbb{Z}$-coefficients to the bi-relative topological cyclic homology groups with $\mathbb{Z} / p^{v} \mathbb{Z}$-coefficients. Hence, in view of Theorem $\mathbb{C}$ we obtain the following stronger result.

Theorem D. Let $f: A \rightarrow B$ be a map of unital associative rings, let $I \subset A$ be a two-sided ideal, and assume that $f: I \rightarrow f(I)$ is an isomorphism onto a two-sided ideal of B. Suppose that the prime number $p$ is nilpotent in A. Then for every integer $q$, the cyclotomic trace map

$$
K_{q}(A, B, I) \rightarrow\left\{\mathrm{TC}_{q}^{n}(A, B, I ; p)\right\}
$$

is an isomorphism of pro-abelian groups. The pro-abelian group on the right-hand side is indexed by positive integers $n$. 
We again remark that Theorem $\mathrm{D}$ is equivalent to the statement that, for $n$ large, the group $K_{q}(A, B, I)$ embeds as a direct summand in $\mathrm{TC}_{q}^{n}(A, B, I ; p)$ and that the system of cokernels satisfies the Mittag-Leffler condition and has limit zero.

Finally, we mention that the main motivation for the work reported in this paper was the application of Theorems $\mathrm{B}$ and $\mathrm{D}$ to the proof in [12, Thm. B] that the mapping fiber of the cyclotomic trace map $K(X) \rightarrow\left\{\mathrm{TC}^{n}(X ; p)\right\}$ satisfies descent for the cdh-topology on the category of schemes essentially of finite type over an infinite perfect field $k$ of positive characteristic $p$, provided that the resolution of singularities holds over $k$. The main advantage of the functor $\left\{\mathrm{TC}_{q}^{n}(-; p)\right\}$ that appears in the statement of Theorems B and $\mathrm{D}$ in comparison to the functor $\mathrm{TC}_{q}(-; p)$ that appears in McCarthy's theorem [23] is that the former preserves filtered colimits while the latter, in general, does not. Therefore, replacing the latter functor by the former, the methods of sheaf cohomology become available.

By a pro-object in a category $\mathscr{C}$, we mean a functor to $\mathscr{C}$ from the set of positive integers viewed as a category with one arrow from $n+1$ to $n$, and by a strict map between pro-objects, we mean a natural transformation. A general map from the pro-object $X=\left\{X_{n}\right\}$ to the pro-object $Y=\left\{Y_{n}\right\}$ is an element of the set

$$
\operatorname{Hom}_{\text {pro }-\mathscr{C}}(X, Y)=\lim _{n} \operatorname{colim}_{m} \operatorname{Hom}_{\mathscr{C}}\left(X_{m}, Y_{n}\right) \text {. }
$$

The category $\mathscr{C}$ embeds in pro $-\mathscr{C}$ as the full subcategory of constant functors. If $\mathscr{C}$ is abelian, then also pro- $\mathscr{C}$ is abelian, and the object $X=\left\{X_{n}\right\}$ is null if and only if for all $n$, there exists $m \geqslant n$ such that the structure map $X_{m} \rightarrow X_{n}$ is zero.

\section{NON-CONNECTIVE $K$-TheORY AND THE CYClOTOMIC TRACE MAP}

In this section, we show that the cyclotomic trace map extends to a map from the Bass completed non-connective $K$-theory to topological cyclic homology. We first briefly review topological cyclic homology and the cyclotomic trace map and refer to [21, Sect. 1] and [16] for the details.

Let $A$ be a unital associative ring. The topological Hochschild spectrum $T(A)$ is a symmetric orthogonal $\mathbb{T}$-spectrum, where $\mathbb{T}$ is the multiplicative group of complex numbers of modulus 1 . Let $p$ be a prime number and let $C_{p^{n-1}} \subset \mathbb{T}$ be the subgroup of the indicated order. We define

$$
\operatorname{TR}^{n}(A ; p)=F\left(\left(\mathbb{T} / C_{p^{n-1}}\right)_{+}, T(A)\right)^{\mathbb{T}}
$$

to be the fixed point spectrum of the function $\mathbb{T}$-spectrum $F\left(\left(\mathbb{T} / C_{p^{n-1}}\right)_{+}, T(A)\right)$. Its homotopy groups are the equivariant homotopy groups

$$
\operatorname{TR}_{q}^{n}(A ; p)=\left[S^{q} \wedge\left(\mathbb{T} / C_{p^{n-1}}\right)_{+}, T(A)\right]_{\mathbb{T}} .
$$

There are two commuting maps of symmetric orthogonal spectra

$$
R, F: \mathrm{TR}^{n}(A ; p) \rightarrow \mathrm{TR}^{n-1}(A ; p)
$$

called the restriction and Frobenius maps. The symmetric orthogonal spectrum $\operatorname{TC}^{n}(A ; p)$ is defined to be the homotopy equalizer of the maps $R$ and $F$, and the topological cyclic homology spectrum is defined to be the homotopy limit

$$
\mathrm{TC}(A ; p)=\operatorname{holim} \operatorname{TC}^{n}(A ; p)
$$

with the structure maps induced by the restriction maps. The homotopy groups of $\mathrm{TC}(A ; p)$ and the $\mathrm{TC}^{n}(A ; p)$ are related by the Milnor exact sequence

$$
0 \rightarrow R^{1} \lim _{n} \mathrm{TC}_{q+1}^{n}(A ; p) \rightarrow \mathrm{TC}_{q}^{n}(A ; p) \rightarrow \lim _{n} \mathrm{TC}_{q}^{n}(A ; p) \rightarrow 0 .
$$


We also consider the homotopy limits

$$
\begin{aligned}
& \operatorname{TR}(A ; p)=\underset{R}{\operatorname{holim}} \operatorname{TR}^{n}(A ; p), \\
& \operatorname{TF}(A ; p)=\underset{F}{\operatorname{holim}} \operatorname{TR}^{n}(A ; p)
\end{aligned}
$$

of the spectra $\operatorname{TR}^{n}(A ; p)$ with respect to the restriction and Frobenius maps, respectively. The Frobenius map induces a self-map of $\operatorname{TR}(A ; p)$, and $\operatorname{TC}(A ; p)$ is canonically isomorphic to the homotopy equalizer of this map and the identity map. Similarly, the restriction map induces a self-map of $\operatorname{TF}(A ; p)$ and $\operatorname{TC}(A ; p)$ is canonically isomorphic to the homotopy equalizer of this map and the identity map. In particular, we have the following long exact sequences of homotopy groups:

$$
\begin{aligned}
& \cdots \rightarrow \mathrm{TC}_{q}(A ; p) \rightarrow \mathrm{TR}_{q}(A ; p) \stackrel{\mathrm{id}-F}{\longrightarrow} \mathrm{TR}_{q}(A ; p) \stackrel{\partial}{\rightarrow} \mathrm{TC}_{q-1}(A ; p) \rightarrow \cdots, \\
& \cdots \rightarrow \mathrm{TC}_{q}(A ; p) \rightarrow \mathrm{TF}_{q}(A ; p) \stackrel{R-\mathrm{id}}{\longrightarrow} \mathrm{TF}_{q}(A ; p) \stackrel{\partial}{\rightarrow} \mathrm{TC}_{q-1}(A ; p) \rightarrow \cdots .
\end{aligned}
$$

It was proved in [20, Thm. $\mathrm{F}$ ] that if the ring $A$ is an algebra over the commutative ring $k$, then the equivariant homotopy groups $\operatorname{TR}_{q}^{n}(A ; p)$ are modules over the ring $W_{n}(k)$ of Witt vectors of length $n$ in $k$.

Lemma 1.1. Let $A$ be a unital associative ring and suppose that the prime number $p$ is nilpotent in $A$. Then for all integers $q$ and $n \geqslant 1$, the groups $\operatorname{TR}_{q}^{n}(A ; p)$ and $\mathrm{TC}_{q}^{n}(A ; p)$ are p-primary torsion groups of bounded exponent.

Proof. Suppose that $A$ is a $\mathbb{Z} / p^{N} \mathbb{Z}$-algebra. Then [20, Thm. F, Prop. 2.7.1] shows that the groups $\operatorname{TR}_{q}^{n}(A ; p)$ are $W_{n}\left(\mathbb{Z} / p^{N} \mathbb{Z}\right)$-modules, and therefore, are annihilated by multiplication by $p^{N n}$. Finally, the long exact sequence

$$
\cdots \longrightarrow \mathrm{TR}_{q+1}^{n-1}(A ; p) \stackrel{\partial}{\longrightarrow} \mathrm{TC}_{q}^{n}(A ; p) \longrightarrow \mathrm{TR}_{q}^{n}(A ; p) \stackrel{R-F}{\longrightarrow} \mathrm{TR}_{q}^{n-1}(A ; p) \longrightarrow \cdots
$$

shows that $\operatorname{TC}_{q}^{n}(A ; p)$ is annihilated by $p^{N(2 n-1)}$.

In general, the groups $\mathrm{TR}_{q}(A ; p)$ and $\mathrm{TC}_{q}(A ; p)$ are not $p$-primary torsion groups of bounded exponent. For example, $\mathrm{TC}_{0}\left(\mathbb{F}_{p} ; p\right)=\mathrm{TR}_{0}\left(\mathbb{F}_{p} ; p\right)=\mathbb{Z}_{p}$.

We consider the diagram of canonical inclusions

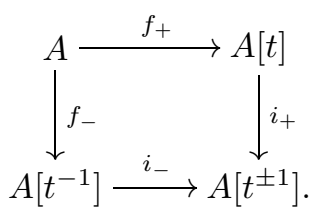

The following result shows that $\operatorname{TR}_{*}^{n}(-; p)$ is a Bass complete theory.

Proposition 1.2. Let $A$ be a unital associative ring. Then for all prime numbers $p$, all integers $q$, and all positive integers $n$, there is an exact sequence

$$
0 \rightarrow \operatorname{TR}_{q}^{n}(A ; p) \rightarrow \underset{\operatorname{TR}_{q}^{n}\left(A\left[t^{-1}\right] ; p\right)}{\operatorname{TR}_{q}^{n}(A[t] ; p)} \rightarrow \operatorname{TR}_{q}^{n}\left(A\left[t^{ \pm 1}\right] ; p\right) \stackrel{\partial_{t}}{\longrightarrow} \operatorname{TR}_{q-1}^{n}(A ; p) \rightarrow 0
$$

where the left-hand map is $\left(f_{+}^{*},-f_{-}^{*}\right)$, and where the middle map is $i_{+}^{*}+i_{-}^{*}$. In addition, the right-hand map $\partial_{t}$ has a section given by multiplication by the image $d \log [t]_{n}$ under the cyclotomic trace map of $t \in K_{1}\left(\mathbb{Z}\left[t^{ \pm 1}\right]\right)$. 
Proof. Multiplication by $d \log [t]_{n}$ defines a map of spectra

$$
\alpha: \Sigma \operatorname{TR}^{n}(A ; p) \rightarrow \operatorname{TR}^{n}\left(A\left[t^{ \pm 1}\right] ; p\right),
$$

and for later use, we remark that $R \alpha=\alpha R$ and $F \alpha=\alpha F$. Now, the proposition is equivalent to the statement that the following sequence is short exact:

$$
\begin{aligned}
0 \rightarrow \mathrm{TR}_{q}^{n}(A ; p) & \rightarrow \mathrm{TR}_{q}^{n}(A[t] ; p) \oplus \mathrm{TR}_{q}^{n}\left(A\left[t^{-1}\right] ; p\right) \oplus \operatorname{TR}_{q-1}^{n}(A ; p) \\
& \rightarrow \mathrm{TR}_{q}^{n}\left(A\left[t^{ \pm 1}\right] ; p\right) \rightarrow 0 .
\end{aligned}
$$

Here the upper map is $\left(f_{+}^{*},-f_{-}^{*}, 0\right)$ and the lower map is $i_{+}^{*}+i_{-}^{*}+\alpha^{*}$. We first prove that the sequence becomes exact after localizing at $p$. Let $r_{+}: A[t] \rightarrow A$ and $r_{-}: A\left[t^{-1}\right] \rightarrow A$ be the ring homomorphisms that map $t$ and $t^{-1}$ to 0 . Since $r_{+} \circ f_{+}$ and $r_{-} \circ f_{-}$are the identity maps, we obtain direct sum decompositions

$$
\begin{aligned}
\operatorname{TR}_{q}^{n}(A[t] ; p) & =\operatorname{TR}_{q}^{n}(A ; p) \oplus N^{+} \operatorname{TR}_{q}^{n}(A ; p), \\
\operatorname{TR}_{q}^{n}\left(A\left[t^{-1}\right] ; p\right) & =\operatorname{TR}_{q}^{n}(A ; p) \oplus N^{-} \operatorname{TR}_{q}^{n}(A ; p) .
\end{aligned}
$$

The structure of the relative terms $N^{+} \operatorname{TR}_{q}^{n}(A ; p)$ and $N^{-} \operatorname{TR}_{q}^{n}(A ; p)$ was determined in [22, Thm. B]. The proof given in loc. cit. also leads to a formula for the groups $\operatorname{TR}_{q}^{n}\left(A\left[t^{ \pm 1}\right] ; p\right)$; we refer to [18, Thm. 2] for the precise statement. By comparing the two formulas, we find that the map

$$
\operatorname{TR}_{q}^{n}(A ; p) \oplus \operatorname{TR}_{q-1}^{n}(A ; p) \oplus N^{+} \operatorname{TR}_{q}^{n}(A ; p) \oplus N^{-} \operatorname{TR}_{q}^{n}(A ; p) \rightarrow \operatorname{TR}_{q}^{n}\left(A\left[t^{ \pm 1}\right] ; p\right)
$$

that, on the first summand, is the map $\iota$ induced by the $A$-algebra homomorphism $i_{+} \circ f_{+}=i_{-} \circ f_{-}$, on the second summand by $\alpha_{*}$, and, on the third and fourth summands, is the compositions of the canonical inclusions of $N^{+} \operatorname{TR}_{q}^{n}(A ; p)$ and $N^{-} \mathrm{TR}_{q}^{n}(A ; p)$ in $\operatorname{TR}_{q}^{n}(A[t] ; p)$ and $\operatorname{TR}_{q}^{n}\left(A\left[t^{-1}\right] ; p\right)$ and the maps induced by the ring homomorphisms $i_{+}$and $i_{-}$, respectively, becomes an isomorphism after localizing at $p$. This shows that the sequence above becomes exact after localizing at $p$.

To prove that the sequence above becomes exact after localizing away from $p$, we recall from [19, Prop. 4.2.5] that for every unital associative ring $B$, the map

$$
\left(R^{n-1-s} F^{s}\right): \operatorname{TR}_{q}^{n}(B ; p) \rightarrow \prod_{0 \leqslant s<n} \operatorname{TR}_{q}^{1}(B ; p)
$$

becomes an isomorphism after localization away from $p$. Therefore, it suffices to consider the case $n=1$. In this case, the description of $\operatorname{TR}_{q}^{n}(A[t] ; p), \operatorname{TR}_{q}^{n}\left(A\left[t^{-1}\right] ; p\right)$, and $\operatorname{TR}_{q}^{n}\left(A\left[t^{ \pm 1}\right] ; p\right)$ that we recalled above is valid without localizing at $p$; compare [22, Lemma 3.3.1]. This completes the proof.

Remark 1.3. The definition of the groups $N \operatorname{TR}_{q}^{n}(A ; p)$ that appear in the proof of Proposition 1.2 is completely analogous to that of the groups $N K_{q}(A)$. However, apart from the definition, there are significant differences between these groups. On the one hand, after localization at $p$, the formula in [22, Thm. B] gives a complete and explicit description of the groups $N \mathrm{TR}_{q}^{n}(A ; p)$ in terms of the groups $\operatorname{TR}_{q}^{n}(A ; p)$, whereas the groups $N K_{q}(A)$ generally do not admit a description in terms of the groups $K_{q}(A)$. On the other hand, the groups $N K_{q}(A)$ vanish if the $\operatorname{ring} A$ is (left) regular, whereas the groups $N \operatorname{TR}_{q}^{n}(A ; p)$ vanish only if $A$ is the zero ring. 
In general, the sequence of Quillen $K$-groups

$$
0 \rightarrow K_{q}(A) \rightarrow \underset{K_{q}(A[t])}{\stackrel{\oplus}{K_{q}\left(A\left[t^{-1}\right]\right)}} \rightarrow K_{q}\left(A\left[t^{ \pm 1}\right]\right) \stackrel{\partial_{t}}{\rightarrow} K_{q-1}(A) \rightarrow 0
$$

is exact only for positive integers $q$, and the Bass negative $K$-groups are recursively defined so as to make the sequence exact for all integers $q$ [1, Chap. XII, Sect. 7]. We recall from Thomason-Trobaugh [26, Def. 6.4] that the Bass completion can be accomplished on the level of spectra. This gives a natural transformation

$$
c: K^{Q}(A) \rightarrow K^{B}(A)
$$

from the Quillen $K$-theory spectrum $K^{Q}(A)$ to a new spectrum $K^{B}(A)$ such that the induced map of homotopy groups is an isomorphism in non-negative degrees and such that the homotopy groups of $K^{B}(A)$ in negative degrees are canonically isomorphic to the Bass negative $K$-groups. We may similarly apply the Bass completion of Thomason-Trobaugh to the topological cyclic homology functor.

Corollary 1.4. The Bass completion map

$$
c: \mathrm{TC}_{q}(A ; p) \rightarrow \mathrm{TC}_{q}^{B}(A ; p)
$$

is an isomorphism for all integers $q$.

Proof. Proposition 1.2 and the Milnor exact sequence show that the sequence

$$
0 \rightarrow \operatorname{TR}_{q}(A ; p) \rightarrow \underset{\operatorname{TR}_{q}\left(A\left[t^{-1}\right] ; p\right)}{\operatorname{TR}_{q}(A[t] ; p)} \rightarrow \operatorname{TR}_{q}\left(A\left[t^{ \pm 1}\right] ; p\right) \stackrel{\partial_{t}}{\rightarrow} \operatorname{TR}_{q-1}(A ; p) \rightarrow 0
$$

is exact for all integers $q$ and that the right-hand map $\partial_{t}$ has a section given by multiplication by the image $d \log [t]$ under the cyclotomic trace of $t \in K_{1}\left(\mathbb{Z}\left[t^{ \pm 1}\right]\right)$. This implies that the Bass completion map

$$
c: \operatorname{TR}_{q}(A ; p) \rightarrow \operatorname{TR}_{q}^{B}(A ; p)
$$

is an isomorphism for all integers $q$. This, in turn, implies that the Bass completion map of the statement is an isomorphism for all integers $q$.

The cyclotomic trace map is a natural map of symmetric spectra

$$
\operatorname{tr}=\operatorname{tr}_{A}: K^{Q}(A) \rightarrow \mathrm{TC}(A ; p) .
$$

It was originally defined by Bökstedt-Hsiang-Madsen [2, but a technically better construction was later given by Dundas-McCarthy [7, Sect. 2.0]. Using the latter construction, we showed in [9, Appendix] that for a commutative $\operatorname{ring} k, \operatorname{tr}_{k}$ is a map of graded rings, and that for an algebra $A$ over a commutative ring $k, \operatorname{tr}_{A}$ is a $\operatorname{tr}_{k}$-linear map of graded modules. Since the Bass completion of [26, Def. 6.4] is functorial, we obtain a commutative diagram of natural transformations

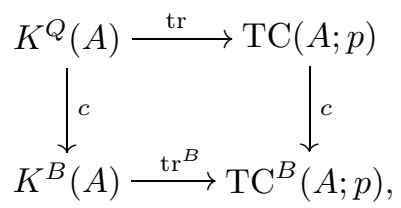

where the lower horizontal map is the map of Bass completed theories induced by the cyclotomic trace, and where, by Corollary 1.4 the right-hand vertical map is a weak equivalence. This gives the desired extension of the cyclotomic trace 
map to a map from the non-connective Bass completed $K$-theory spectrum to the Bass completed topological cyclic homology spectrum. In the following, we do not distinguish the topological cyclic homology spectrum and its Bass completion, and we write

$$
\operatorname{tr}: K(A) \rightarrow \mathrm{TC}(A ; p)
$$

for the lower horizontal map in the diagram above.

\section{The Relative THEOREM}

The proofs of Theorems $\mathrm{A}$ and $\mathrm{B}$ are based on the description in [15, Sect. 2] of the topological Hochschild $\mathbb{T}$-spectrum of split square zero extensions of rings. We briefly recall this description.

We let $B$ be a unital associative ring, and let $I$ be a $B$ - $B$-bimodule. Then the ring $A=B \ltimes I$ defined by the product abelian group $B \times I$ with multiplication

$$
(b, x) \cdot\left(b^{\prime}, x^{\prime}\right)=\left(b b^{\prime}, b x^{\prime}+x b^{\prime}\right)
$$

is a split square zero extension of the $\operatorname{ring} B$ by the ideal $I$, and up to isomorphism, every split square zero extension of rings is of this form. In this situation, we recall from [15, Prop. 2.1] that there is a wedge decomposition of $\mathbb{T}$-spectra

$$
\bigvee_{r \geqslant 1} T(B \ltimes I ; r) \stackrel{\sim}{\longrightarrow} T(A, I),
$$

where the wedge sum ranges over the set of positive integers. We remark that the spectrum $T(B \ltimes I ; r)$ was denoted $T_{r}(B \oplus I)$ in op. cit. We write the induced direct sum decomposition of equivariant homotopy groups as

$$
\bigoplus_{r \geqslant 1} \mathrm{TR}_{q}^{n}(B \ltimes I ; r ; p) \stackrel{\sim}{\rightarrow} \mathrm{TR}_{q}^{n}(A, I ; p) .
$$

The Frobenius map $F: \operatorname{TR}_{q}^{n}(A, I ; p) \rightarrow \mathrm{TR}_{q}^{n-1}(A, I ; p)$ preserves the direct sum decomposition while the restriction map $R: \operatorname{TR}_{q}^{n}(A, I ; p) \rightarrow \mathrm{TR}_{q}^{n-1}(A, I ; p)$ maps the summand indexed by the positive integer $r$ divisible by $p$ to the summand indexed by $r / p$ and annihilates the remaining summands. Moreover, it follows from [20, Thm. 2.2] that there is a long exact sequence

$$
\cdots \rightarrow \mathbb{H}_{q}\left(C_{p^{n-1}}, T(B \ltimes I ; r)\right) \rightarrow \mathrm{TR}_{q}^{n}(B \ltimes I ; r ; p) \stackrel{R}{\rightarrow} \mathrm{TR}_{q}^{n-1}(B \ltimes I ; r / p ; p) \rightarrow \cdots,
$$

where the right-hand group is understood to be zero if $p$ does not divide $r$. The left-hand group is the $q$ th group homology of $C_{p^{n-1}}$ with coefficients in $T(B \ltimes I ; r)$ and is the abutment of the strongly convergent spectral sequence

$$
E_{s, t}^{2}=H_{s}\left(C_{p^{n-1}}, \mathrm{TR}_{t}^{1}(B \ltimes I ; r ; p)\right) \Rightarrow \mathbb{H}_{s+t}\left(C_{p^{n-1}}, T(B \ltimes I ; r)\right) ;
$$

see e.g. [18, Sect. 4]. By the definition of the $\mathbb{T}$-spectrum $T(B \ltimes I ; r)$, the homotopy groups $\mathrm{TR}_{t}^{1}(B \ltimes I ; r ; p)=\pi_{t} T(B \ltimes I ; r)$ are zero for $t<r-1$. Hence, the spectral sequence and long exact sequence above imply that the restriction map

$$
R: \operatorname{TR}_{q}^{n}(B \ltimes I ; r ; p) \rightarrow \mathrm{TR}_{q}^{n-1}(B \ltimes I ; r / p ; p)
$$

is an isomorphism for $q<r-1$, and an epimorphism for $q=r-1$. In particular, for every $n$ and $q$, only finitely many summands in the direct sum decomposition of the group $\operatorname{TR}_{q}^{n}(A, I ; p)$ are non-zero. 
Lemma 2.1. Let $p$ be a prime number, and let $X$ be a spectrum whose homotopy groups are p-primary torsion groups of bounded exponent. Then for all integers $q$, the canonical map defines an isomorphism of pro-abelian groups

$$
\pi_{q}(X) \stackrel{\sim}{\rightarrow}\left\{\pi_{q}\left(X, \mathbb{Z} / p^{v} \mathbb{Z}\right)\right\} .
$$

Here $\pi_{q}\left(X, \mathbb{Z} / p^{v} \mathbb{Z}\right)$ denotes the qth homotopy groups with $\mathbb{Z} / p^{v} \mathbb{Z}$-coefficients, and the pro-abelian group on the right-hand side is indexed by positive integers $v$.

Proof. The coefficient sequences give a short exact sequence of pro-abelian groups

$$
0 \rightarrow\left\{\pi_{q}(X) \otimes \mathbb{Z} / p^{v} \mathbb{Z}\right\} \rightarrow\left\{\pi_{q}\left(X, \mathbb{Z} / p^{v} \mathbb{Z}\right)\right\} \rightarrow\left\{\operatorname{Tor}_{1}^{\mathbb{Z}}\left(\pi_{q-1}(X), \mathbb{Z} / p^{v} \mathbb{Z}\right)\right\} \rightarrow 0,
$$

and the map of the statement factors through the canonical projection

$$
\pi_{q}(X) \rightarrow\left\{\pi_{q}(X) \otimes \mathbb{Z} / p^{v} \mathbb{Z}\right\} .
$$

The latter map is an isomorphism of pro-abelian groups, since the structure maps in the target pro-abelian group are isomorphisms if $p^{v}$ is larger than the exponent of $\pi_{q}(X)$. Similarly, the pro-abelian group on the right-hand side in the short exact sequence above is isomorphic to zero, since the structure map

$$
\operatorname{Tor}_{1}^{\mathbb{Z}}\left(\pi_{q-1}(X), \mathbb{Z} / p^{2 v} \mathbb{Z}\right) \rightarrow \operatorname{Tor}_{1}^{\mathbb{Z}}\left(\pi_{q-1}(X), \mathbb{Z} / p^{v} \mathbb{Z}\right)
$$

is zero if $p^{v}$ is larger than the exponent of $\pi_{q-1}(X)$.

Theorem 2.2. Let $A$ be a unital associative ring and $I \subset A$ a two-sided nilpotent ideal. Suppose that the prime number $p$ is nilpotent in $A$. Then for every integer $q$, the canonical map defines an isomorphism of pro-abelian groups

$$
\mathrm{TC}_{q}(A, I ; p) \stackrel{\sim}{\rightarrow}\left\{\mathrm{TC}_{q}^{n}(A, I ; p)\right\} .
$$

Proof. We consider the following diagram of pro-abelian groups in which the maps are the canonical maps:

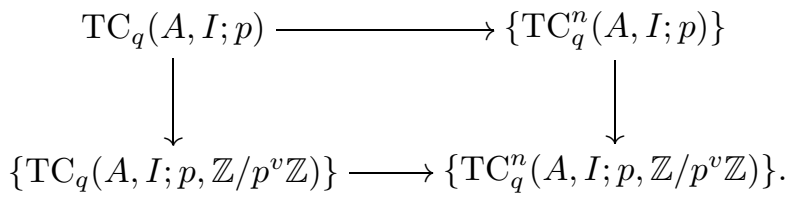

We have previously proved in [11, Thm. 2.1.1] that the the lower horizontal map is an isomorphism. Moreover, by Lemma 1.1, the groups $\mathrm{TC}_{q}^{n}(A, I ; p)$ are $p$-primary torsion groups of bounded exponent, and hence Lemma 2.1 shows that the righthand vertical map is an isomorphism. Therefore, the statement of the theorem is equivalent to the statement that the left-hand vertical map is an isomorphism. Hence, by Lemma 2.1, it will suffice to show that the groups $\mathrm{TC}_{q}(A, I ; p)$ are $p$ primary torsion groups of bounded exponent.

We first assume that $I \subset A$ is a square zero ideal and that the canonical projection of $A$ onto the quotient $\operatorname{ring} B=A / I$ admits a ring section. We use the wedge decomposition of $T(A, I)$ which we recalled above to give a formula for the spectrum $\operatorname{TC}(A, I ; p)$. First, for the homotopy limit with respect to $F$, we find

$$
\begin{aligned}
\operatorname{TF}(A, I ; p) & =\underset{F}{\operatorname{holim}} \operatorname{TR}^{n}(A, I ; p) \stackrel{\sim}{\leftarrow} \operatorname{holim}_{F} \bigvee_{r \geqslant 1} \operatorname{TR}^{n}(B \ltimes I ; r ; p) \\
& \stackrel{\sim}{\rightarrow} \prod_{r \geqslant 1} \operatorname{holim}_{F} \operatorname{TR}^{n}(B \ltimes I ; r ; p)=\prod_{r \geqslant 1} \operatorname{TF}(B \ltimes I ; r ; p),
\end{aligned}
$$


where the second map is a weak equivalence since, for every $n$ and $q$, the homotopy groups $\operatorname{TR}_{q}^{n}(B \ltimes I ; r ; p)$ are non-zero for only finitely many $r$. Writing $r=p^{v-1} j$, we may rewrite the product on the right-hand side as

$$
\prod_{r \geqslant 1} \mathrm{TF}(B \ltimes I ; r ; p)=\prod_{j \in I_{p}} \prod_{v \geqslant 0} \mathrm{TF}\left(B \ltimes I ; p^{v-1} j ; p\right),
$$

where $I_{p}$ denotes the set of positive integers not divisible by $p$. Then the restriction map takes the factor indexed by $(j, v)$ to the factor indexed by $(j, v-1)$. Hence, taking the homotopy equalizer of the restriction map and the identity map, we obtain a weak equivalence

$$
\mathrm{TC}(A, I ; p) \simeq \prod_{j \in I_{p}} \operatorname{holim} \operatorname{TF}\left(B \ltimes I ; p^{v-1} j ; p\right) .
$$

We argued in the discussion preceding Lemma 2.1 that the restriction map

$$
R: \operatorname{TR}_{q}^{n}\left(B \ltimes I ; p^{v-1} j ; p\right) \rightarrow \mathrm{TR}_{q}^{n-1}\left(B \ltimes I ; p^{v-2} j ; p\right)
$$

is an isomorphism if $q+1<p^{v-1} j$. Hence, the Milnor exact sequence shows that for $1 \leqslant j \leqslant q+1$, the canonical projection induces an isomorphism

$$
\pi_{q}\left(\underset{R}{\operatorname{holim}} \operatorname{TF}\left(B \ltimes I ; p^{v-1} j ; p\right)\right) \stackrel{\sim}{\rightarrow} \operatorname{TF}_{q}\left(B \ltimes I ; p^{s-1} j ; p\right),
$$

where $s=s_{p}(q, j)$ is the unique integer such that $p^{s-1} j \leqslant q+1<p^{s} j$, and that for $q+1<j$, the homotopy group on the left-hand side vanishes. Therefore, to show that $\mathrm{TC}_{q}(A, I ; p)$ is a $p$-primary torsion group of bounded exponent, it suffices to show that for all integers $q$ and $s \geqslant 1$ and all $j \in I_{p}, \mathrm{TF}_{q}\left(B \ltimes I ; p^{s-1} j ; p\right)$ is a $p$-primary torsion group of bounded exponent. Now, from [20, Thm. 2.2], we have the following cofibration sequence of spectra:

$$
\begin{aligned}
& \mathbb{H} .\left(C_{p^{n-1}}, T\left(B \ltimes I ; p^{s-1} j\right)\right) \rightarrow \mathrm{TR}^{n}\left(B \ltimes I ; p^{s-1} j ; p\right) \\
& \stackrel{R}{\rightarrow} \mathrm{TR}^{n-1}\left(B \ltimes I ; p^{s-2} j ; p\right) \stackrel{\partial}{\rightarrow} \Sigma \mathbb{H} .\left(C_{p^{n-1}}, T\left(B \ltimes I ; p^{s-1} j\right)\right) .
\end{aligned}
$$

The Frobenius maps induce maps between these cofibration sequences, and taking the homotopy limit, we obtain the following cofibration sequence of spectra:

$$
\begin{aligned}
& \underset{F}{\operatorname{holim}} \mathbb{H} .\left(C_{p^{n-1}}, T\left(B \ltimes I ; p^{s-1} j\right)\right) \rightarrow \operatorname{TF}\left(B \ltimes I ; p^{s-1} j ; p\right) \\
& \stackrel{R}{\rightarrow} \operatorname{TF}\left(B \ltimes I ; p^{s-2} j ; p\right) \stackrel{\partial}{\rightarrow} \sum \underset{F}{\operatorname{holim}} \mathbb{H} .\left(C_{p^{n-1}}, T\left(B \ltimes I ; p^{s-1} j\right)\right) .
\end{aligned}
$$

Hence, by induction on $s \geqslant 1$, it will suffice to prove that for all $s \geqslant 1$ and $j \in I_{p}$, the homotopy groups of the left-hand term are $p$-primary torsion groups of bounded exponent. We write $r=p^{s-1} j$ and recall the spectral sequence

$$
E_{s, t}^{2}=H_{s}\left(C_{p^{n-1}}, \operatorname{TR}_{t}^{1}(B \ltimes I ; r ; p)\right) \Rightarrow \mathbb{H}_{s+t}\left(C_{p^{n-1}}, T(B \ltimes I ; r ; p)\right) ;
$$

see [18, Sect. 4] for a detailed discussion. Since the spectral sequence induces a finite filtration of the abutment, we obtain a spectral sequence of pro-abelian groups

$$
\left\{E_{s, t}^{2}\right\}=\left\{H_{s}\left(C_{p^{n-1}}, \mathrm{TR}_{t}^{1}(B \ltimes I ; r ; p)\right)\right\} \Rightarrow\left\{\mathbb{H}_{s+t}\left(C_{p^{n-1}}, T(B \ltimes I ; r ; p)\right)\right\},
$$

where the pro-abelian groups are indexed by integers $n \geqslant 1$, and where the structure maps in the pro-abelian groups are the Frobenius maps. Since the $C_{p^{n-1} \text {-action on }}$ the spectrum $\mathrm{TR}^{1}(B \ltimes I ; r ; p)$ is obtained from a $\mathbb{T}$-action, the induced action on the homotopy groups $\operatorname{TR}_{t}^{1}(B \ltimes I ; r ; p)$ is trivial. Moreover, the homotopy groups $\operatorname{TR}_{t}^{1}(B \ltimes I ; r ; p)$ are annihilated by the same power of $p$ that annihilates $B$. It follows 
that for $n$ large, $\mathbb{H}_{s}\left(C_{p^{n-1}}, \mathrm{TR}_{t}^{1}(B \ltimes I ; r ; p)\right)$ is isomorphic to $\mathrm{TR}_{t}^{1}(B \ltimes I ; r ; p)$. On $E^{2}$-terms, the Frobenius map induces the transfer map in group homology

$$
F: H_{s}\left(C_{p^{n-1}}, \mathrm{TR}_{t}^{1}(B \ltimes I ; r ; p)\right) \rightarrow H_{s}\left(C_{p^{n-2}}, \mathrm{TR}_{t}^{1}(B \ltimes I ; r ; p)\right)
$$

which is readily evaluated [18, Lemma 6]. We conclude that there are isomorphisms of pro-abelian groups

$$
\left\{E_{s, t}^{2}\right\} \cong \begin{cases}\mathrm{TR}_{t}^{1}(B \ltimes I ; r ; p) & (s \text { odd }), \\ 0 & (s \text { even }) .\end{cases}
$$

Therefore, the spectral sequence above shows that for all integers $q$, the pro-abelian group $\left\{\mathbb{H}_{q}\left(C_{p^{n-1}}, T(B \ltimes I ; r)\right)\right\}$ is isomorphic to a constant pro-abelian group and that this constant pro-abelian group is a $p$-primary torsion group of bounded exponent. But then the canonical map

$$
\pi_{q}\left(\operatorname{holim} \mathbb{F} \mathbb{H} .\left(C_{p^{n-1}}, T(B \ltimes I ; r)\right)\right) \rightarrow\left\{\mathbb{H}_{q}\left(C_{p^{n-1}}, T(B \ltimes I ; r)\right)\right\}
$$

is an isomorphism of pro-abelian groups, and hence also the left-hand group is a $p$-primary torsion group of bounded exponent as desired. This completes the proof of the theorem in the case where $A$ is a split square zero extension.

We next let $I \subset A$ be any square zero ideal and show that for all integers $q$, the pro-abelian group $\left\{\mathrm{TC}_{q}^{n}(A, I ; p)\right\}$ is isomorphic to a $p$-primary torsion group of bounded exponent. It then follows from the Milnor exact sequence that the map of the statement is an isomorphism of pro-abelian groups. We first choose a weak equivalence of simplicial rings

$$
\epsilon_{A / I}: A / I[-] \rightarrow A / I
$$

such that the ring $A / I[k]$ is a free unital associative ring for all $k \geqslant 0$. Then the map $\epsilon_{A}: A[-] \rightarrow A$ defined by the pull-back diagram of simplicial rings

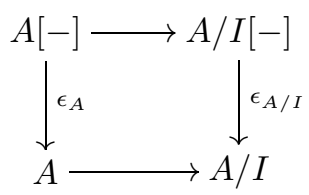

again is a weak equivalence. In this case, there is a spectral sequence

$$
E_{s, t}^{1}=\mathrm{TC}_{t}^{n}(A[s], I ; p) \Rightarrow \mathrm{TC}_{s+t}^{n}(A, I ; p),
$$

for every integer $n \geqslant 1$; compare the proof of Lemma 3.8 below. Since the spectral sequence is concentrated in the first quadrant, we obtain, as $n$ varies, a spectral sequence of pro-abelian groups

$$
\left\{E_{s, t}^{1}\right\}=\left\{\mathrm{TC}_{t}^{n}(A[s], I ; p)\right\} \Rightarrow\left\{\mathrm{TC}_{s+t}^{n}(A, I ; p)\right\}
$$

Moreover, since $A / I[k]$ is a free unital associative ring, the canonical projection from $A[k]$ onto $A / I[k]$ admits a ring section. Therefore, by the case considered earlier, we conclude that for all integers $s$ and $t$, the pro-abelian group $\left\{E_{s, t}^{1}\right\}$ is isomorphic to a constant pro-abelian group. This implies that for all integers $q$, the pro-abelian group $\left\{\mathrm{TC}_{q}^{n}(A, I ; p)\right\}$ is isomorphic to a constant pro-abelian group. Indeed, the category of constant pro-abelian groups is a full abelian subcategory of the abelian category of all pro-abelian groups. This completes the proof in the case where $I \subset A$ is a square zero ideal. 
Finally, let $I \subset A$ be a two-sided ideal such that $I^{m}=0$. We show by induction on $m \geqslant 2$ that the map of the statement is an isomorphism of pro-abelian groups. The case $m=2$ was proved above, so we let $m>2$ and assume that the statement has been proved for smaller $m$. We consider the long exact sequence

$$
\cdots \rightarrow \mathrm{TC}_{q}\left(A, I^{m-1} ; p\right) \rightarrow \mathrm{TC}_{q}(A, I ; p) \rightarrow \mathrm{TC}_{q}\left(A / I^{m-1}, I / I^{m-1} ; p\right) \rightarrow \cdots .
$$

By induction, the right-hand and left-hand groups both are $p$-torsion groups of bounded exponents, and therefore, so is the middle group. This completes the proof.

Proof of Theorem [A. The arithmetic square [3, Prop. 2.9] gives rise to the following long exact sequence where the two products range over all prime numbers:

$$
\cdots \longrightarrow K_{q}(A, I) \longrightarrow \underset{K_{q}(A, I) \otimes \mathbb{Q}}{\oplus} \longrightarrow\left(\prod_{\ell} K_{q}\left(A, I ; \mathbb{Z}_{\ell}\right)\right) \otimes \mathbb{Q} \longrightarrow \cdots
$$

The theorem of Goodwillie [14, Main Thm.] identifies $K_{q}(A, I) \otimes \mathbb{Q}$ with the relative negative cyclic homology group $\mathrm{HC}_{q}^{-}(A \otimes \mathbb{Q}, I \otimes \mathbb{Q})$ which, in turn, is zero, since $p$ is nilpotent in $A$. Similarly, McCarthy's theorem [23, Main Thm.] shows that for every prime number $\ell$, the cyclotomic trace map induces an isomorphism

$$
K_{q}\left(A, I ; \mathbb{Z}_{\ell}\right) \stackrel{\sim}{\rightarrow} \mathrm{TC}_{q}\left(A, I ; \ell, \mathbb{Z}_{\ell}\right) .
$$

Since $p$ is nilpotent in $A$, this group vanishes for $\ell \neq p$ by Lemma 1.1. Moreover, Theorem 2.2 implies that the completion map $\operatorname{TC}_{q}(A, I ; p) \rightarrow \mathrm{TC}_{q}\left(A, I ; p, \mathbb{Z}_{p}\right)$ is an isomorphism and that the common group is a $p$-primary torsion group of bounded exponent. We conclude that the cyclotomic trace is an isomorphism

$$
K_{q}(A, I) \stackrel{\sim}{\rightarrow} \mathrm{TC}_{q}(A, I ; p)
$$

and that $K_{q}(A, I)$ is a $p$-primary torsion group of bounded exponent as stated.

Proof of Theorem B. The map of the statement is equal to the composition

$$
K_{q}(A, I) \rightarrow \mathrm{TC}_{q}(A, I ; p) \rightarrow\left\{\mathrm{TC}_{q}^{n}(A, I ; p)\right\}
$$

of the cyclotomic trace map and the canonical map. We saw in the proof of Theorem A above that the former map is an isomorphism, and we proved in Theorem 2.2 that the latter map is an isomorphism. The theorem follows.

\section{THE BI-RELATIVE THEOREM}

In this section, we prove Theorems $[\mathrm{C}$ and $\mathrm{D}$ of the introduction. We view the ideal $I$ as an associative ring without unit and form the associated associative ring with unit $\mathbb{Z} \ltimes I$ defined to be the product abelian group $\mathbb{Z} \times I$ with multiplication

$$
(a, x) \cdot\left(a^{\prime}, x^{\prime}\right)=\left(a a^{\prime}, a x^{\prime}+x a^{\prime}+x x^{\prime}\right) .
$$

We recall that Suslin has proved in $\left[25\right.$, Thm. A] that if $\operatorname{Tor}_{q}^{\mathbb{Z} \ltimes I}(\mathbb{Z}, \mathbb{Z})$ vanishes for all $q>0$, then the bi-relative group $K_{q}(A, B, I)$ vanishes for all $q$. We prove the following result in a similar manner. 
Theorem 3.1. Let $f: A \rightarrow B$ be a map of unital associative rings, let $I \subset A$ be a two-sided ideal and assume that $f: I \rightarrow f(I)$ is an isomorphism onto a two-sided ideal of B. Suppose that the pro-abelian group $\left\{\operatorname{Tor}_{q}^{\mathbb{Z} \ltimes\left(I^{m}\right)}(\mathbb{Z}, \mathbb{Z})\right\}$ is zero for all positive integers $q$. Then the canonical map

$$
K_{q}(A, B, I) \rightarrow\left\{K_{q}\left(A / I^{m}, B / I^{m}, I / I^{m}\right)\right\}
$$

is an isomorphism of pro-abelian groups for all integers $q$.

Proof. We have a long exact sequence of pro-abelian groups

$$
\cdots \rightarrow\left\{K_{q}\left(A, B, I^{m}\right)\right\} \rightarrow K_{q}(A, B, I) \rightarrow\left\{K_{q}\left(A / I^{m}, B / I^{m}, I / I^{m}\right)\right\} \rightarrow \cdots
$$

and must show that the left-hand term is zero for all integers $q$. Let $\overline{G L}\left(A / I^{m}\right)$ be the image of the map $G L(A) \rightarrow G L\left(A / I^{m}\right)$ induced by the canonical projection. The mapping fiber $F\left(A, I^{m}\right)$ of the induced map $B G L(A)^{+} \rightarrow B \overline{G L}\left(A / I^{m}\right)^{+}$is a connected space with $\pi_{q}\left(F\left(A, I^{m}\right)\right)=K_{q}\left(A, I^{m}\right)$ for $q \geqslant 1$. Since $K_{q}\left(A, B, I^{m}\right)$ vanishes for $q \leqslant 0$ by [1, Thm. XII.8.3], it will suffice to show that the map

$$
\left\{\pi_{q}\left(F\left(A, I^{m}\right)\right)\right\} \rightarrow\left\{\pi_{q}\left(F\left(B, I^{m}\right)\right)\right\}
$$

induced by $f$ is an isomorphism of pro-abelian groups for all $q \geqslant 1$. Now, the spaces $F\left(A, I^{m}\right)$ and $F\left(B, I^{m}\right)$ are $H$-spaces and hence simple. Therefore, by the generalized Whitehead theorem [24, Cor. 4.3], it will suffice to show that the map

$$
\left\{H_{q}\left(F\left(A, I^{m}\right) ; \mathbb{Z}\right)\right\} \rightarrow\left\{H_{q}\left(F\left(B, I^{m}\right) ; \mathbb{Z}\right)\right\}
$$

induced by $f$ is an isomorphism of pro-abelian groups for all $q \geqslant 1$. The proof of this, in turn, is completely analogous to the (rather long) proof of [10, Prop. 1.3].

Remark 3.2. We do not know whether or not the canonical map

$$
K_{q}(A, B, I) \rightarrow\left\{K_{q}\left(A / I^{m}, B / I^{m}, I / I^{m}\right)\right\}
$$

is an isomorphism of pro-abelian groups if the assumption that the pro-abelian groups $\left\{\operatorname{Tor}_{q}^{\mathbb{Z} \ltimes\left(I^{m}\right)}(\mathbb{Z}, \mathbb{Z})\right\}$ be zero is omitted.

We proceed to show in Proposition 3.7 below that the hypotheses of Theorem 3.1 are satisfied if the ideal $I$ can be embedded as an ideal of a free unital $\mathbb{F}_{p^{-}}$ algebra. The proof relies on the non-standard homological algebra developed in Suslin's paper [25], or rather a slight generalization thereof, which we first discuss.

Let $k$ be a commutative ring and let $\left\{I_{m}\right\}$ be a pro-non-unital associative $k$ algebra. We define a left $\left\{I_{m}\right\}$-module to be a pro- $k$-module $\left\{M_{m}\right\}$ together with a structure of a left $I_{m}$-module on $M_{m}$ for every $m \geqslant 1$ such that the diagrams

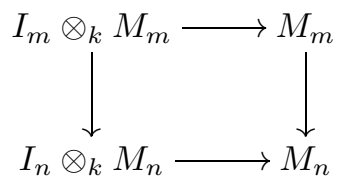

commute. Here the horizontal maps are the module structure maps and the vertical maps are induced from the structure maps in the pro- $k$-modules $\left\{I_{m}\right\}$ and $\left\{M_{m}\right\}$. A homomorphism from the left $\left\{I_{m}\right\}$-module $\left\{M_{m}\right\}$ to the left $\left\{I_{m}\right\}$-module $\left\{M_{m}^{\prime}\right\}$ is defined to be a strict map of pro- $k$-modules $f:\left\{M_{m}\right\} \rightarrow\left\{M_{m}^{\prime}\right\}$ such that the map $f_{m}: M_{m} \rightarrow M_{m}^{\prime}$ is $I_{m}$-linear for all $m \geqslant 1$. An extended left $\left\{I_{m}\right\}$-module is a left $\left\{I_{m}\right\}$-module of the form $\left\{I_{m} \otimes_{k} L_{m}\right\}$, where $\left\{L_{m}\right\}$ is a pro-k-module. The left $\left\{I_{m}\right\}$-module $\left\{P_{m}\right\}$ is pseudo-free if there exists an isomorphism of left 
$\left\{I_{m}\right\}$-modules $\varphi:\left\{I_{m} \otimes_{k} L_{m}\right\} \rightarrow\left\{P_{m}\right\}$ from an extended left $\left\{I_{m}\right\}$-module such that each $L_{m}$ is a free $k$-module. The homomorphism $f:\left\{P_{m}\right\} \rightarrow\left\{M_{m}\right\}$ from the pseudo-free left $\left\{I_{m}\right\}$-module $\left\{P_{m}\right\}$ to the left $\left\{I_{m}\right\}$-module $\left\{M_{m}\right\}$ is special if there exists an isomorphism $\varphi:\left\{I_{m} \otimes_{k} L_{m}\right\} \rightarrow\left\{P_{m}\right\}$ with each $L_{m}$ free and a strict map of pro- $k$-modules $g:\left\{L_{m}\right\} \rightarrow\left\{M_{m}\right\}$ such that the diagrams

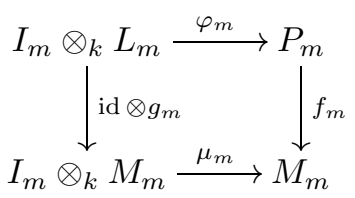

commute. Here the lower horizontal map is the module structure map.

Lemma 3.3. Let $k$ be a commutative ring and $\left\{I_{m}\right\}$ a pro-non-unital associative $k$-algebra. Then for all $q \geqslant 0$, the special homomorphism $f:\left\{P_{m}\right\} \rightarrow\left\{M_{m}\right\}$ from the pseudo-free left $\left\{I_{m}\right\}$-module $\left\{P_{m}\right\}$ to the left $\left\{I_{m}\right\}$-module $\left\{M_{m}\right\}$ induces the zero map of pro- $k$-modules

$$
\left\{\operatorname{Tor}_{q}^{k \ltimes I_{m}}\left(k, P_{m}\right)\right\} \rightarrow\left\{\operatorname{Tor}_{q}^{k \ltimes I_{m}}\left(k, M_{m}\right)\right\} .
$$

Proof. There exists a commutative diagram

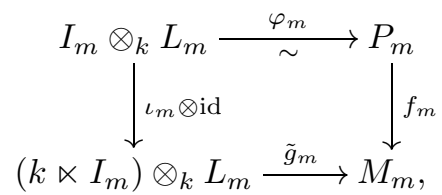

where $\tilde{g}_{m}((a, x) \otimes y)=a g_{m}(y)+x g_{m}(y)$. For $q=0$, the map $\iota_{m}$ induces the zero map $k \otimes_{k \ltimes I_{m}} I_{m} \rightarrow k \otimes_{k \ltimes I_{m}}\left(k \ltimes I_{m}\right)$, and for $q>0$,

$$
\operatorname{Tor}_{q}^{k \ltimes I_{m}}\left(k,\left(k \ltimes I_{m}\right) \otimes L_{m}\right)=\operatorname{Tor}_{q}^{k \ltimes I_{m}}\left(k, k \ltimes I_{m}\right) \otimes L_{m}=0 .
$$

The lemma follows.

Proposition 3.4. Let $k$ be a commutative ring, let $\left\{I_{m}\right\}$ be a pro-non-unital associative $k$-algebra, and assume that for all $q>0$, the pro-k-module

$$
\left\{\operatorname{Tor}_{q}^{k \ltimes I_{m}}(k, k)\right\}
$$

is zero. Let $F_{q}, q \geqslant 0$, be functors from the category of left $\left\{I_{m}\right\}$-modules to the category of pro-abelian groups and assume that the following (i)-(ii) hold.

(i) If $\left\{M_{m}[-]\right\} \rightarrow\left\{M_{m}\right\}$ is an augmented simplicial left $\left\{I_{m}\right\}$-module such that the associated chain complex of pro-abelian groups is exact, then there is a spectral sequence of pro-abelian groups

$$
E_{s, t}^{1}=F_{t}\left(\left\{M_{m}[s]\right\}\right) \Rightarrow F_{s+t}\left(\left\{M_{m}\right\}\right)
$$

whose edge-homomorphism $F_{t}\left(\left\{M_{m}[0]\right\}\right) \rightarrow F_{t}\left(\left\{M_{m}\right\}\right)$ is equal to the map induced by the augmentation $\epsilon:\left\{M_{m}[0]\right\} \rightarrow\left\{M_{m}\right\}$.

(ii) If $f:\left\{P_{m}\right\} \rightarrow\left\{M_{m}\right\}$ is a special homomorphism, then for all $q \geqslant 0$, the induced map of pro-abelian groups $f_{*}: F_{q}\left(\left\{P_{m}\right\}\right) \rightarrow F_{q}\left(\left\{M_{m}\right\}\right)$ is zero.

Then for every pseudo-free left $\left\{I_{m}\right\}$-module $\left\{P_{m}\right\}$ and every $q \geqslant 0$, the pro-abelian group $F_{q}\left(\left\{P_{m}\right\}\right)$ is zero. 
Proof. This is proved as in [10, Prop. 1.7] by induction on $q \geqslant 0$. The additional assumption in loc. cit. that for every left $\left\{I_{m}\right\}$-module $\left\{M_{m}\right\}$, the pro-abelian group $F_{0}\left(\left\{M_{m}\right\}\right)$ be zero is unnecessary. Indeed, the proof of the induction step given in loc. cit. also proves the case $q=0$.

We remark that, in Proposition 3.4, the functors $F_{q}$ are not assumed to be additive.

Let $\alpha: k^{\prime} \rightarrow k$ be a ring homomorphism and let $\left\{I_{m}\right\}$ be a pro-non-unital associative $k$-algebra. We define $\left\{I_{m}^{\alpha}\right\}$ to be $\left\{I_{m}\right\}$ considered as a pro-non-unital associative $k^{\prime}$-algebra via $\alpha$ and call it the associated pro-non-unital associative $k^{\prime}$-algebra. Moreover, if $\left\{M_{m}\right\}$ is a left $\left\{I_{m}\right\}$-module, we define $\left\{M_{m}^{\alpha}\right\}$ to be $\left\{M_{m}\right\}$ considered as a left $\left\{I_{m}^{\alpha}\right\}$-module via $\alpha$ and call it the associated left $\left\{I_{m}^{\alpha}\right\}$-module.

Lemma 3.5. Let $\left\{I_{m}\right\}$ be a pro-non-unital associative $k$-algebra, let $\left\{P_{m}\right\}$ be a pseudo-free left $\left\{I_{m}\right\}$-module, let $f:\left\{P_{m}\right\} \rightarrow\left\{M_{m}\right\}$ be a special homomorphism, and suppose that $\alpha: k^{\prime} \rightarrow k$ is a surjective ring homomorphism. Then $\left\{P_{m}^{\alpha}\right\}$ is a pseudo-free left $\left\{I_{m}^{\alpha}\right\}$-module and $f:\left\{P_{m}^{\alpha}\right\} \rightarrow\left\{M_{m}^{\alpha}\right\}$ is a special homomorphism.

Proof. Let $\varphi:\left\{I_{m} \otimes_{k} L_{m}\right\} \rightarrow\left\{P_{m}\right\}$ be an isomorphism such that each $L_{m}$ is a free $k$-module. For every $m$, we choose a free $k^{\prime}$-module $L_{m}^{\prime}$ and an isomorphism of $k$-modules $\psi_{m}: k \otimes_{k^{\prime}} L_{m}^{\prime} \rightarrow L_{m}$. We define $\varphi_{m}^{\prime}: I_{m} \otimes_{k^{\prime}} L_{m}^{\prime} \rightarrow P_{m}$ to be the isomorphism of left $I_{m}$-modules defined by the composition

$$
I_{m} \otimes_{k^{\prime}} L_{m}^{\prime} \rightarrow I_{m} \otimes_{k} k \otimes_{k^{\prime}} L_{m}^{\prime} \rightarrow I_{m} \otimes_{k} L_{m} \rightarrow P_{m}
$$

of the canonical isomorphism and the isomorphisms id $\otimes \psi_{m}$ and $\varphi_{m}$. Now, let $h_{m}: L_{m+1} \rightarrow L_{m}$ be the structure map in the pro- $k$-module $\left\{L_{m}\right\}$. Then there exist a $k^{\prime}$-linear map $h_{m}^{\prime}: L_{m+1}^{\prime} \rightarrow L_{m}^{\prime}$ that makes the diagram

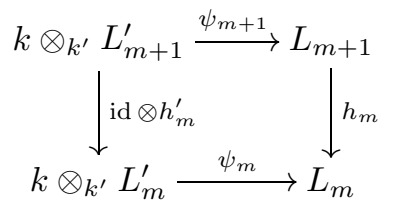

commute. Indeed, this follows from the surjectivity of the following composite map:

$$
\begin{aligned}
& \operatorname{Hom}_{k^{\prime}}\left(L_{m+1}^{\prime}, L_{m}^{\prime}\right) \rightarrow \operatorname{Hom}_{k^{\prime}}\left(L_{m+1}^{\prime}, k \otimes_{k^{\prime}} L_{m}^{\prime}\right) \\
& \rightarrow \operatorname{Hom}_{k^{\prime}}\left(L_{m+1}^{\prime}, L_{m}\right) \rightarrow \operatorname{Hom}_{k}\left(k \otimes_{k^{\prime}} L_{m+1}^{\prime}, L_{m}\right) .
\end{aligned}
$$

Here the first map is induced by the canonical map and is surjective, since $\alpha: k^{\prime} \rightarrow k$ is surjective and since $L_{m+1}^{\prime}$ is free, and hence projective; the second map is the isomorphism induced by $\psi_{m}$; the third map is the canonical isomorphism. We now define $\left\{L_{m}^{\prime}\right\}$ to be the pro- $k^{\prime}$-module with the maps $h_{m}^{\prime}: L_{m+1}^{\prime} \rightarrow L_{m}^{\prime}$ as the structure maps. Then the map $\varphi^{\prime}:\left\{I_{m}^{\alpha} \otimes_{k^{\prime}} L_{m}^{\prime}\right\} \rightarrow\left\{P_{m}^{\alpha}\right\}$ is an isomorphism of left $\left\{I_{m}^{\alpha}\right\}$-modules, and hence, $\left\{P_{m}^{\alpha}\right\}$ is a pseudo-free left $\left\{I_{m}^{\alpha}\right\}$-module as stated.

Finally, let $g:\left\{L_{m}\right\} \rightarrow\left\{M_{m}\right\}$ be a map of pro- $k$-modules such that the diagram

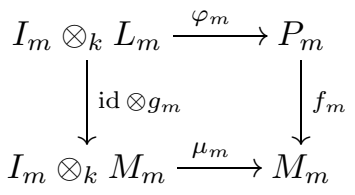


commutes. We define $g_{m}^{\prime}: L_{m}^{\prime} \rightarrow M_{m}^{\alpha}$ to be the $k^{\prime}$-linear map given by the composition of the canonical map $L_{m}^{\prime} \rightarrow k \otimes_{k^{\prime}} L_{m}^{\prime}$, the isomorphism $\psi_{m}$, and the map $g_{m}$. Then the map $g^{\prime}:\left\{L_{m}^{\prime}\right\} \rightarrow\left\{M_{m}^{\alpha}\right\}$ is a map of pro- $k^{\prime}$-modules and the diagrams

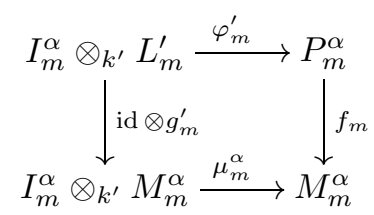

commute. This shows that $f:\left\{P_{m}^{\alpha}\right\} \rightarrow\left\{M_{m}^{\alpha}\right\}$ is special as stated.

Proposition 3.6. Let $\alpha: k^{\prime} \rightarrow k$ be a surjective map of commutative rings, let $\left\{I_{m}\right\}$ a pro-non-unital associative $k$-algebra, and let $\left\{I_{m}^{\alpha}\right\}$ be the associated pro-non-unital associative $k^{\prime}$-algebra. Assume that the pro-k-module

$$
\left\{\operatorname{Tor}_{q}^{k \ltimes I_{m}}(k, k)\right\}
$$

is zero for all integers $q>0$. Then, the pro-k'-module

$$
\left\{\operatorname{Tor}_{q}^{k^{\prime} \ltimes I_{m}^{\alpha}}\left(k^{\prime}, k^{\prime}\right)\right\}
$$

is zero for all integers $q>0$.

Proof. The short exact sequence of left $k^{\prime} \ltimes I_{m}^{\alpha}$-modules

$$
0 \rightarrow I_{m}^{\alpha} \rightarrow k^{\prime} \ltimes I_{m}^{\alpha} \rightarrow k^{\prime} \rightarrow 0
$$

induces a long exact sequence of pro-abelian groups

$$
\cdots \rightarrow\left\{\operatorname{Tor}_{q}^{k^{\prime} \ltimes I_{m}^{\alpha}}\left(k^{\prime}, I_{m}^{\alpha}\right)\right\} \rightarrow\left\{\operatorname{Tor}_{q}^{k^{\prime} \ltimes I_{m}^{\alpha}}\left(k^{\prime}, k^{\prime} \ltimes I_{m}^{\alpha}\right)\right\} \rightarrow\left\{\operatorname{Tor}_{q}^{k^{\prime} \ltimes I_{m}^{\alpha}}\left(k^{\prime}, k^{\prime}\right)\right\} \rightarrow \cdots .
$$

For $q>0$, the middle term is zero, and for $q=0$, the left-hand map is zero. Hence, for all $q>0$, the boundary map is an isomorphism of pro-abelian groups

$$
\left\{\operatorname{Tor}_{q}^{k^{\prime} \ltimes I_{m}^{\alpha}}\left(k^{\prime}, k^{\prime}\right)\right\} \stackrel{\sim}{\longrightarrow}\left\{\operatorname{Tor}_{q-1}^{k^{\prime} \ltimes I_{m}^{\alpha}}\left(k^{\prime}, I_{m}^{\alpha}\right)\right\} .
$$

Therefore, the statement will follow from Proposition 3.4 once we verify that the following functors $F_{q}, q \geqslant 0$, from the category of left $\left\{I_{m}\right\}$-modules to the category of pro-abelian groups satisfy the hypotheses (i)-(ii) of loc. cit.:

$$
F_{q}\left(\left\{M_{m}\right\}\right)=\left\{\operatorname{Tor}_{q}^{k^{\prime} \ltimes I_{m}^{\alpha}}\left(k^{\prime}, M_{m}^{\alpha}\right)\right\} .
$$

Indeed, considered as a left $\left\{I_{m}\right\}$-module, $\left\{I_{m}\right\}$ is pseudo-free.

To prove hypothesis (i), we let $\epsilon:\left\{M_{m}[-]\right\} \rightarrow\left\{M_{m}\right\}$ be an augmented simplicial left $\left\{I_{m}\right\}$-module and consider the bi-simplicial symmetric spectrum

$$
X[s, t]=B\left(H k^{\prime}, H\left(k^{\prime} \ltimes I_{m}^{\alpha}\right), H M_{m}^{\alpha}[s]\right)[t]=H k^{\prime} \wedge H\left(k^{\prime} \ltimes I_{m}^{\alpha}\right)^{\wedge t} \wedge H M_{m}^{\alpha}[s]
$$

given by the two-sided bar-construction of the Eilenberg-Mac Lane spectra associated with the ring $k^{\prime} \ltimes I_{m}^{\alpha}$ and the left and right modules $k^{\prime}$ and $M_{m}^{\alpha}[s]$. We recall that the three possible ways of forming the geometric realization of $X[-,-]$ lead to the same result in the sense that there are canonical isomorphisms

$$
|[s] \mapsto|[t] \mapsto X[s, t]|\stackrel{\sim}{\longrightarrow}|[n] \mapsto X[n, n]|\stackrel{\sim}{\leftarrow}|[t] \mapsto|[s] \mapsto X[s, t]| \mid .
$$

For the left-hand side, the skeleton filtration gives rise to a spectral sequence that converges to the homotopy groups of the geometric realization. By [8, Thm. 2.1], we obtain the identification

$$
E_{s, t}^{1}=\pi_{t}(|[i] \mapsto X[s, i]|)=\operatorname{Tor}_{t}^{k^{\prime} \ltimes I_{m}^{\alpha}}\left(k^{\prime}, M_{m}^{\alpha}[s]\right) .
$$


For the right-hand side, the augmentation $\epsilon$ induces a weak equivalence

$$
|[s] \mapsto X[s, t]| \stackrel{\sim}{\rightarrow} B\left(H k^{\prime}, H\left(k^{\prime} \ltimes I_{m}^{\alpha}\right), H M_{m}^{\alpha}\right)[t]
$$

for each $t$. By [13, Prop. VII.3.6], the induced map of realizations

$$
|[t] \mapsto|[s] \mapsto X[s, t]|\stackrel{\sim}{\rightarrow}|[t] \mapsto B\left(H k^{\prime}, H\left(k^{\prime} \ltimes I_{m}^{\alpha}\right), H M_{m}^{\alpha}\right)[t] \mid .
$$

is a weak equivalence. We conclude from [8, Thm. 2.1] that

$$
\pi_{q}(|[t] \mapsto|[s] \mapsto X[s, t]||)=\operatorname{Tor}_{q}^{k^{\prime} \ltimes I_{m}^{\alpha}}\left(k^{\prime}, M_{m}^{\alpha}\right) .
$$

Hence, we have a spectral sequence

$$
E_{s, t}^{1}=\operatorname{Tor}_{t}^{k^{\prime} \ltimes I_{m}^{\alpha}}\left(k^{\prime}, M_{m}^{\alpha}[s]\right) \Rightarrow \operatorname{Tor}_{s+t}^{k^{\prime} \ltimes I_{m}^{\alpha}}\left(k^{\prime}, M_{m}^{\alpha}\right),
$$

which proves hypothesis (i).

To prove hypothesis (ii), let $f:\left\{P_{m}\right\} \rightarrow\left\{M_{m}\right\}$ be a special homomorphism of left $\left\{I_{m}\right\}$-modules. Then Lemma 3.5 shows that $f:\left\{P_{m}^{\alpha}\right\} \rightarrow\left\{M_{m}^{\alpha}\right\}$ is a special homomorphism of left $\left\{I_{m}^{\alpha}\right\}$-modules, and hence, Lemma 3.3 shows that the induced map $f_{*}: F_{q}\left(\left\{P_{m}\right\}\right) \rightarrow F_{q}\left(\left\{M_{m}\right\}\right)$ is zero for $q \geqslant 0$, as required.

Proposition 3.7. Let I be a two-sided ideal in a free unital associative $\mathbb{F}_{p}$-algebra. Then for all positive integers $q$, the following pro-abelian group is zero:

$$
\left\{\operatorname{Tor}_{q}^{\mathbb{Z} \ltimes\left(I^{m}\right)}(\mathbb{Z}, \mathbb{Z})\right\} .
$$

Proof. By Proposition [3.6, it suffices to show that for all $q>0$, the pro-abelian group

$$
\left\{\operatorname{Tor}_{q}^{\mathbb{F}_{p} \ltimes\left(I^{m}\right)}\left(\mathbb{F}_{p}, \mathbb{F}_{p}\right)\right\}
$$

is zero. Since $\mathbb{F}_{p}$ is a field, the Tor-groups in question are canonically isomorphic to the homology groups of the normalized bar-complex

$$
B\left(\mathbb{F}_{p}, \mathbb{F}_{p} \ltimes I^{m}, \mathbb{F}_{p}\right)=\mathbb{F}_{p} \otimes_{\mathbb{F}_{p} \ltimes I^{m}} B\left(\mathbb{F}_{p} \ltimes I^{m}, \mathbb{F}_{p}\right),
$$

which takes the form

$$
\cdots \stackrel{d_{m}}{\longrightarrow}\left(I^{m}\right)^{\otimes n} \stackrel{d_{m}}{\longrightarrow} \cdots \stackrel{d_{m}}{\longrightarrow}\left(I^{m}\right)^{\otimes 2} \stackrel{d_{m}}{\longrightarrow} I^{m} \stackrel{d_{m}}{\longrightarrow} \mathbb{F}_{p}
$$

with differential

$$
d_{m}\left(x_{1} \otimes \cdots \otimes x_{n}\right)=\sum_{i=1}^{n-1}(-1)^{i} x_{1} \otimes \cdots \otimes x_{i} x_{i+1} \otimes \cdots \otimes x_{n} .
$$

We define a chain homotopy from the chain map

$$
\iota: B\left(\mathbb{F}_{p}, \mathbb{F}_{p} \ltimes I^{2 m}, \mathbb{F}_{p}\right) \rightarrow B\left(\mathbb{F}_{p}, \mathbb{F}_{p} \ltimes I^{m}, \mathbb{F}_{p}\right)
$$

induced by the canonical inclusion to the chain map that is zero in positive degrees and the identity map in degree 0 . Replacing $I$ by $I^{m}$, we may assume that $m=1$. Suppose that $I$ is a two-sided ideal in the free unital associative $\mathbb{F}_{p}$-algebra $F$. Then, as a left $F$-module, $I$ is free and hence projective [4, Cor. 2.4.3]. It follows that the multiplication $\mu: F \otimes I \rightarrow I$ has an $F$-linear section $\alpha: I \rightarrow F \otimes I$. We remark that $\alpha$ restricts to an $I$-linear map $\alpha: I^{2} \rightarrow I \otimes I$. Then

$$
s\left(x_{1} \otimes \cdots \otimes x_{n}\right)=(-1)^{n} x_{1} \otimes \cdots \otimes x_{n-1} \otimes \alpha\left(x_{n}\right)
$$


is the desired chain-homotopy. We conclude that for $q>0$, the canonical inclusion of $I^{2 m}$ into $I^{m}$ induces the zero map

$$
\operatorname{Tor}_{q}^{\mathbb{F}_{p} \ltimes I^{2 m}}\left(\mathbb{F}_{p}, \mathbb{F}_{p}\right) \rightarrow \operatorname{Tor}_{q}^{\mathbb{F}_{p} \ltimes I^{m}}\left(\mathbb{F}_{p}, \mathbb{F}_{p}\right) .
$$

This completes the proof.

It was proved by Goodwillie [14, Lemma I.2.2] that the relative $K$-theory of a nilpotent extension of simplicial rings may be evaluated degreewise. We need the following analogous result for bi-relative $K$-theory.

Lemma 3.8. Let $f: A \rightarrow B$ be a map of unital associative rings and $I \subset A$ a twosided ideal such that $f: I \rightarrow f(I)$ is an isomorphism onto a two-sided ideal of $B$. Let $f[-]: A[-] \rightarrow B[-]$ be a map of simplicial unital associative rings and $I[-]$ a simplicial two-sided ideal of $A[-]$ such that $f[-]: I[-] \rightarrow f(I[-])$ is an isomorphism onto a simplicial two-sided ideal of $B[-]$. In addition, let $\epsilon_{A}: A[-] \rightarrow A$ and $\epsilon_{B}: B[-] \rightarrow B$ be weak equivalences of simplicial unital associative rings such that $f \circ \epsilon_{A}=\epsilon_{B} \circ f[-]$ and such that $\epsilon_{A}$ restricts to a weak equivalence of simplicial associative rings $\epsilon_{I}: I[-] \rightarrow I$. Then there is a natural spectral sequence

$$
E_{s, t}^{1}=K_{t}(A[s], B[s], I[s]) \Rightarrow K_{s+t}(A, B, I) .
$$

Proof. We have a spectral sequence

$$
E_{s, t}^{1}=K_{t}(A[s], B[s], I[s]) \Rightarrow \pi_{s+t}(|[n] \mapsto K(A[n], B[n], I[n])|)
$$

obtained from the skeleton filtration of the geometric realization. Hence, it suffices to construct a weak equivalence between the symmetric spectrum $K(A, B, I)$ and the symmetric spectrum $|[n] \mapsto K(A[n], B[n], I[n])|$. The definition of $K$-theory was extended to simplicial rings by Waldhausen [27]. Moreover, by op. cit., Prop. 1.1, the extended functor preserves weak equivalence such that, in the case at hand, the augmentations induce a weak equivalence

$$
\epsilon_{*}: K(A[-], B[-], I[-]) \stackrel{\sim}{\rightarrow} K(A, B, I) .
$$

We proceed to relate the left-hand side to the symmetric spectrum given by the geometric realization above. Let $\Delta[n][-]$ be the simplicial standard $n$-simplex whose set of $m$-simplices $\Delta[n][m]$ is the set non-decreasing maps from $[m]$ to $[n]$. Then if $S[-]$ is a simplicial ring, we define $S[-,-]$ to be the bi-simplicial ring

$$
S[m, n]=\operatorname{Hom}(\Delta[m][-] \times \Delta[n][-], S[-]),
$$

where the right-hand side is the set of maps of simplicial sets. For every $n$, the canonical projection gives rise to a map of simplicial rings $\operatorname{pr}_{1}^{*}: S[-] \rightarrow S[-, n]$, which is a weak equivalence. It induces a map of symmetric spectra

$$
\operatorname{pr}_{1}^{*}: K(A[-], B[-], I[-]) \rightarrow|[n] \mapsto K(A[-, n], B[-, n], I[-, n])|,
$$

which is a weak equivalence by [27, Prop. 1.1] and [13, Prop. VII.3.6]. Similarly, we have the map of simplicial rings $\operatorname{pr}_{2}^{*}: S[n] \rightarrow S[-, n]$, where the ring $S[n]$ is considered as a constant simplicial ring, which induces a map of symmetric spectra

$$
\operatorname{pr}_{2}^{*}:|[n] \mapsto K(A[n], B[n], I[n])| \rightarrow|[n] \mapsto K(A[-, n], B[-, n], I[-, n])| .
$$

The composition of the map of homotopy groups induced by $\mathrm{pr}_{2}^{*}$, the inverse of the map of homotopy groups induced by the weak equivalence $\mathrm{pr}_{1}^{*}$, and the map of homotopy groups induced by the weak equivalence $\epsilon_{*}$ defines a natural map

$$
f_{K}: \pi_{q}(|[n] \mapsto K(A[n], B[n], I[n])|) \rightarrow K_{q}(A, B, I),
$$


and the lemma will follow if we prove that this map is an isomorphism.

By the arithmetic square [3, Prop. 2.9], it will suffice to show that the map $f$ induces an isomorphism of rational homotopy groups and, for every prime number $\ell$, an isomorphism of homotopy groups with $\mathbb{Z} / \ell \mathbb{Z}$-coefficients. For the rational homotopy groups, we compare the bi-relative $K$-groups to the corresponding birelative negative cyclic and cyclic homology groups. By [14, Sect. I.3], the latter are also defined for simplicial rings. Hence, we may follow the proceedure above and define maps $f_{\mathrm{HC}^{-}}$and $f_{\mathrm{HC}}$ that make the following diagram commute:

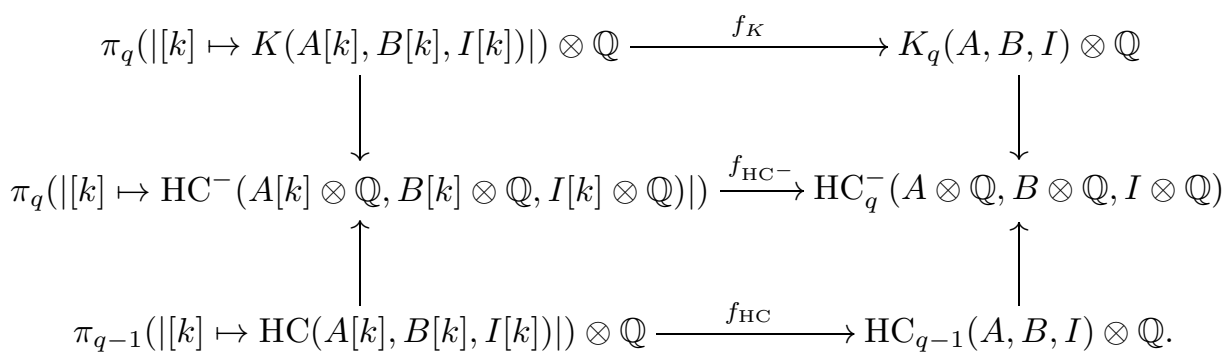

By theorems of Cortiñas [5. Thm. 0.1] and Cuntz-Quillen [6], the vertical maps are isomorphisms. Moreover, it follows immediately from the definition of cyclic homology that the lower horizontal map is an isomorphism. Finally, for the homotopy groups with $\mathbb{Z} / \ell \mathbb{Z}$-coefficients, we compare the bi-relative $K$-groups to the corresponding bi-relative topological cyclic homology groups. The latter are defined for simplicial rings, and hence, we obtain the following commutative diagram:

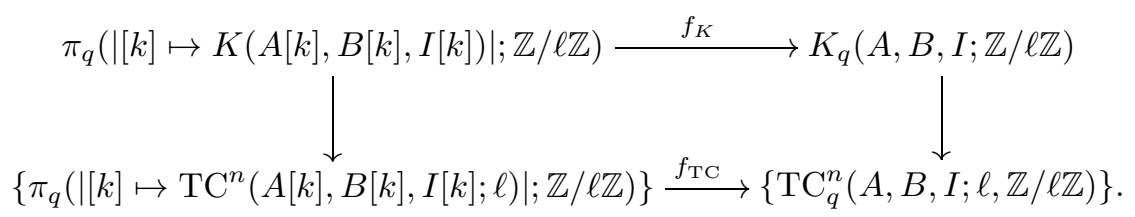

It follows from our previous result [10, Thm. 1] that the vertical maps are isomorphisms of pro-abelian groups. We must show that the lower horizontal map is an isomorphism of pro-abelian groups. We prove the stronger statement that the map of integral homotopy groups

$$
f_{\mathrm{TC}}: \pi_{q}\left(\left|[k] \mapsto \mathrm{TC}^{n}(A[k], B[k], I[k] ; \ell)\right| \rightarrow \mathrm{TC}_{q}^{n}(A, B, I ; \ell)\right.
$$

is an isomorphism for all integers $q$ and $n \geqslant 1$. The cofibration sequence

$$
\mathrm{TC}^{n}(-; \ell) \rightarrow \mathrm{TR}^{n}(-; \ell) \rightarrow \mathrm{TR}^{n-1}(-; \ell) \rightarrow \Sigma \mathrm{TC}^{n}(-; \ell)
$$

shows that it will suffice to show that the map

$$
f_{\mathrm{TR}}: \pi_{q}\left(\left|[k] \mapsto \mathrm{TR}^{n}(A[k], B[k], I[k] ; \ell)\right|\right) \rightarrow \mathrm{TR}_{q}^{n}(A, B, I ; \ell)
$$

is an isomorphism for all integers $q$ and $n \geqslant 1$. We recall the following cofibration sequence from 20, Thm. 2.2]:

$$
\mathbb{H} .\left(C_{\ell^{n-1}}, T(-)\right) \rightarrow \mathrm{TR}^{n}(-; \ell) \rightarrow \mathrm{TR}^{n-1}(-; \ell) \rightarrow \Sigma \mathbb{H} .\left(C_{\ell^{n-1}}, T(-)\right) .
$$

The functor $\mathbb{H}$. $\left(C_{\ell^{n-1}},-\right)$ commutes with geometric realization, up to canonical isomorphism, and preserves weak equivalences. Therefore, it will suffice to show 
that $f_{\mathrm{TR}}$ is an isomorphism for $n=1$ and for all $q$. Finally, the functor $\mathrm{TR}^{1}(-; \ell)$ is the topological Hochschild homology functor $\mathrm{THH}(-)$, and we wish to show that

$$
f_{\mathrm{THH}}: \pi_{q}(|[k] \mapsto \operatorname{THH}(A[k], B[k], I[k])|) \rightarrow \operatorname{THH}_{q}(A, B, I)
$$

is an isomorphism for all integers $q$. This follows immediately from the definition of topological Hochschild homology.

Proof of Theorem $\mathbb{C}$. We first assume that $(A, B, I)$ is a triple of $\mathbb{F}_{p}$-algebras and that $I$ admits an embedding as a two-sided ideal in a free unital associative $\mathbb{F}_{p^{-}}$ algebra. In this case, Proposition 3.7 shows that the pro-abelian group

$$
\left\{\operatorname{Tor}_{q}^{\mathbb{Z} \ltimes\left(I^{m}\right)}(\mathbb{Z}, \mathbb{Z})\right\}
$$

is zero for every $q>0$, and hence, Theorem 3.1 shows that the canonical map

$$
K_{q}(A, B, I) \rightarrow\left\{K_{q}\left(A / I^{m}, B / I^{m}, I / I^{m}\right)\right\}
$$

is an isomorphism of pro-abelian groups. In particular, the group $K_{q}(A, B, I)$ is a direct summand in $K_{q}\left(A / I^{m}, B / I^{m}, I / I^{m}\right)$ for $m$ large. Now, by the definition of the bi-relative groups, we have a long exact sequence

$$
\cdots \rightarrow K_{q}\left(A / I^{m}, B / I^{m}, I / I^{m}\right) \rightarrow K_{q}\left(A / I^{m}, I / I^{m}\right) \rightarrow K_{q}\left(B / I^{m}, I / I^{m}\right) \rightarrow \cdots .
$$

By Theorem $\mathrm{A}$ the middle and right-hand groups are $p$-primary torsion groups of bounded exponent, and hence, the same holds for the left-hand group. This shows that $K_{q}(A, B, I)$ is a $p$-primary torsion group of bounded exponent as stated.

In the general case, we let $N$ be a positive integer such that $p^{N}$ annihilates $A$ and choose a diagram of simplicial unital associative $\mathbb{Z} / p^{N} \mathbb{Z}$-algebras

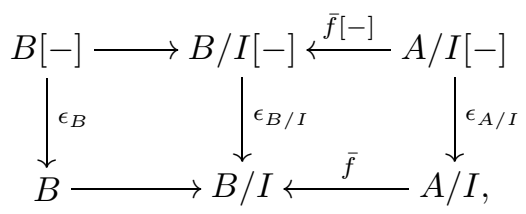

such that the vertical maps are weak equivalences and such that the simplicial algebras in the top row are degree-wise free unital associative $\mathbb{Z} / p^{N} \mathbb{Z}$-algebras. We then consider the diagram of simplicial $\mathbb{Z} / p^{N} \mathbb{Z}$-algebras

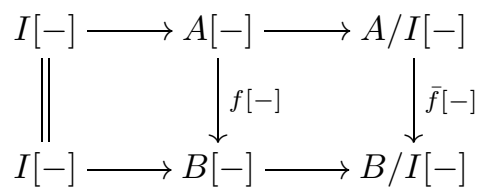

with $A[-]$ defined to be the pull-back of $B[-]$ along $\bar{f}[-]$ and with the left-hand horizontal maps defined to be the kernels of the respective right-hand horizontal maps. The maps $\epsilon_{B}, \epsilon_{B / I}$, and $\epsilon_{A / I}$ above induce maps of simplicial associative $\mathbb{Z} / p^{N} \mathbb{Z}$-algebras $\epsilon_{A}: A[-] \rightarrow A$ and $\epsilon_{I}: I[-] \rightarrow I$ which are weak equivalences. It follows from Lemma 3.8 that there is a spectral sequence

$$
E_{s, t}^{1}=K_{t}(A[s], B[s], I[s]) \Rightarrow K_{s+t}(A, B, I) .
$$

Hence, it suffices to show that $K_{q}(A[s], B[s], I[s])$ is a $p$-primary torsion group of bounded exponent for all integers $q$ and $s \geqslant 0$. Since $B / I[s]$ is a free unital 
associative $\mathbb{Z} / p^{N} \mathbb{Z}$-algebra, the canonical projections of $A[s]$ onto $A / I[s]$ and $B[s]$ onto $B / I[s]$ have ring sections. Therefore, the diagram

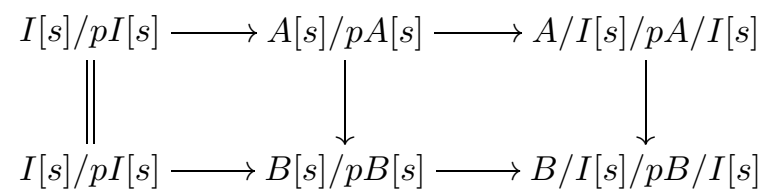

is a diagram of associative $\mathbb{F}_{p}$-algebras in which the right-hand square is a pull-back and the left-hand horizontal maps are the kernels of the right-hand horizontal maps. Now, as $B[s] / p B[s]$ is a free unital associative $\mathbb{F}_{p}$-algebra, the special case considered first shows that $K_{q}(A[s] / p A[s], B[s] / p B[s], I[s] / p I[s])$ is a $p$-primary torsion group of bounded exponent for all integers $q$ and $s \geqslant 0$. Moreover, the mapping fiber of the map induced by the canonical projections

$$
K(A[s], B[s], I[s]) \rightarrow K(A[s] / p A[s], B[s] / p B[s], I[s] / p I[s])
$$

is canonically isomorphic to the iterated mapping fiber of the following square of relative $K$-theory spectra:

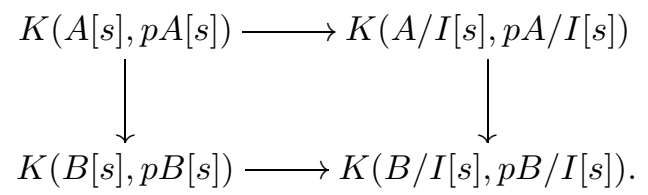

Theorem $\AA$ shows that the homotopy groups of each of the four terms in this square are $p$-primary torsion groups of bounded exponent. Hence, the same is true for the homotopy groups of the iterated mapping fiber. This shows that $K_{q}(A[s], B[s], I[s])$ is a $p$-primary torsion group of bounded exponent for all integers $q$ and $s \geqslant 0$, as desired. The theorem follows.

Proof of Theorem $\mathrm{D}$. We consider the following diagram of pro-abelian groups:

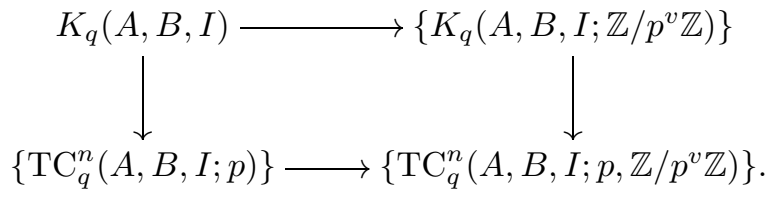

It follows from Theorem $\mathrm{C}$ and Lemma 2.1 that the upper horizontal map is an isomorphism. Similarly, Lemma 2.1 shows that the lower horizontal map is an isomorphism. Finally, we proved in [10, Thm. 1] that the right-hand vertical map is an isomorphism. Hence, the left-hand vertical map is an isomorphism as stated.

\section{REFERENCES}

[1] H. Bass, Algebraic K-theory, W. A. Benjamin, Inc., New York-Amsterdam, 1968. MR0249491 $(40: 2736)$

[2] M. Bökstedt, W.-C. Hsiang, and I. Madsen, The cyclotomic trace and algebraic K-theory of spaces, Invent. Math. 111 (1993), 465-540. MR1202133 (94g:55011)

[3] A. K. Bousfield, The localization of spectra with respect to homology, Topology 18 (1979), 257-281. MR551009 (80m:55006)

[4] P. M. Cohn, Free rings and their relations. Second edition, London Mathematical Society Monographs, vol. 19, Academic Press, Inc., London, 1985. MR800091(87e:16006)

[5] G. Cortiñas, The obstruction to excision in K-theory and in cyclic homology, Invent. Math. 164 (2006), 143-173. MR2207785 (2006k:19006) 
[6] J. Cuntz and D. Quillen, Excision in bivariant periodic cyclic cohomology, Invent. Math. 127 (1997), 67-98. MR1423026 (98g:19003)

[7] B. I. Dundas and R. McCarthy, Topological Hochschild homology of ring functors and exact categories, J. Pure Appl. Alg. 109 (1996), 231-294. MR.1388700(97i:19001)

[8] A. D. Elmendorf, I. Kriz, M. A. Mandell, and J. P. May, Rings, modules, and algebras in stable homotopy theory. With an appedix by M. Cole, Mathematical Surveys and Monographs, vol. 47, Amer. Math. Soc., Providence, RI, 1997. MR1417719 (97h:55006)

[9] T. Geisser and L. Hesselholt, Topological cyclic homology of schemes, K-theory (Seattle, 1997), Proc. Symp. Pure Math., vol. 67, 1999, pp. 41-87. MR1743237(2001g:19003)

[10] B B-relative algebraic K-theory and topological cyclic homology, Invent. Math. 166 (2006), 359-395. MR2249803 (2008a:19003)

[11] _ On the K-theory and topological cyclic homology of smooth schemes over a discrete valuation ring, Trans. Amer. Math. Soc. 358 (2006), 131-145. MR2171226 (2006g:19008)

[12] _ On the vanishing of negative K-groups, Math. Ann. 348 (2010), 707-736.

[13] P. G. Goerss and J. F. Jardine, Simplicial homotopy theory, Progress in Mathematics, vol. 174, Birkhäuser, Boston, MA, 1999. MR1711612(2001d:55012)

[14] T. G. Goodwillie, Relative algebraic K-theory and cyclic homology, Ann. of Math. (2) 124 (1986), 347-402. MR855300(88b:18008)

[15] L. Hesselholt, Stable topological cyclic homology is topological Hochschild homology, K-theory (Strasbourg, 1992), Astérisque, vol. 226, 1994, pp. 175-192. MR1317119 (96b:19004)

[16] _ K-theory of truncated polynomial algebras, Handbook of $K$-theory, Springer-Verlag, New York, 2005, pp. 71-110. MR2181821 (2006m:19005)

[17] _ On the K-theory of the coordinate axes in the plane, Nagoya Math. J. 185 (2007), 93-109. MR2301459 (2008d:19003)

[18] - On the Whitehead spectrum of the circle, Algebraic Topology (Oslo, Norway, 2007), Abel Symp., vol. 4, Springer-Verlag, Berlin, 2009, pp. 131-184. MR2597738

[19] L. Hesselholt and I. Madsen, Cyclic polytopes and the K-theory of truncated polynomial algebras, Invent. Math. 130 (1997), 73-97. MR1471886 (98k:19002)

[20] _ On the K-theory of finite algebras over Witt vectors of perfect fields, Topology 36 (1997), 29-102. MR1410465 (97i:19002)

[21] , On the K-theory of local fields, Ann. of Math. (2) 158 (2003), 1-113. MR.1998478 (2004k:19003)

[22] - On the de Rham-Witt complex in mixed characteristic, Ann. Sci. École Norm. Sup. 37 (2004), 1-43. MR2050204 (2005f:19005)

[23] R. McCarthy, Relative algebraic K-theory and topological cyclic homology, Acta Math. 179 (1997), 197-222. MR 1607555 (99e:19006)

[24] S. Singh, The Dror-Whitehead theorem in pro-homotopy and shape theories, Trans. Amer. Math. Soc. 268 (1981), 487-496. MR632540 (83b:55004)

[25] A. A. Suslin, Excision in the integral algebraic K-theory, Proc. Steklov Inst. Math. 208 (1995), 255-279. MR:1730271 (2000i:19011)

[26] R. W. Thomason and T. Trobaugh, Higher algebraic K-theory of schemes and of derived categories, Grothendieck Festschrift, Volume III, Progress in Mathematics, vol. 88, 1990, pp. 247-435. MR.1106918 (92f:19001)

[27] F. Waldhausen, Algebraic K-theory of topological spaces. I, Algebraic and geometric topology, Proc. Symp. Pure Math., vol. 32, Amer. Math. Soc., Providence, RI, 1978, pp. 35-60. MR.520492(81i:18014a)

[28] C. A. Weibel, Mayer-Vietoris sequences and module structure on $N K_{*}$, Algebraic $K$-theory (Evanston, IL, 1980), Lecture Notes in Math., vol. 854, Springer-Verlag, New York, 1981, pp. 466-493. MR618317 (82k:18010)

Department of Mathematics, University of Southern California, 3620 Vermont Avenue KAP 108, Los Angeles, California 90089

E-mail address: geisser@usc.edu

Graduate School of Mathematics, Nagoya University, Furo-cho, Chikusa-ku, Nagoya, 464-8602 JAPAN

E-mail address: larsh@math.nagoya-u.ac.jp 